See discussions, stats, and author profiles for this publication at: https://www.researchgate.net/publication/272998805

\title{
On the Martingale Representation Theorem and on Approximate Hedging a Contingent Claim in the Minimum D....
}

\section{Chapter · August 2007}

DOI: 10.1142/9789812709356_0008

\section{CITATION}

1

2 authors, including:
READS

16

\section{Quan-Hoang Vuong}

Western University Hanoi (Đại Học Thành Tây), Vietnam 151 PUBLICATIONS 401 CITATIONS

SEE PROFILE

Some of the authors of this publication are also working on these related projects:

The Network of Vietnamese Social Scientists (a Vuong \& Associates research 
Series in Contemporary Applied Mathematics

CAM 8

Some Toniss in Industrial and Apnlied Mathematiss

Rolf Jeltsch Ta-Tsien Li

Ian H Sloan

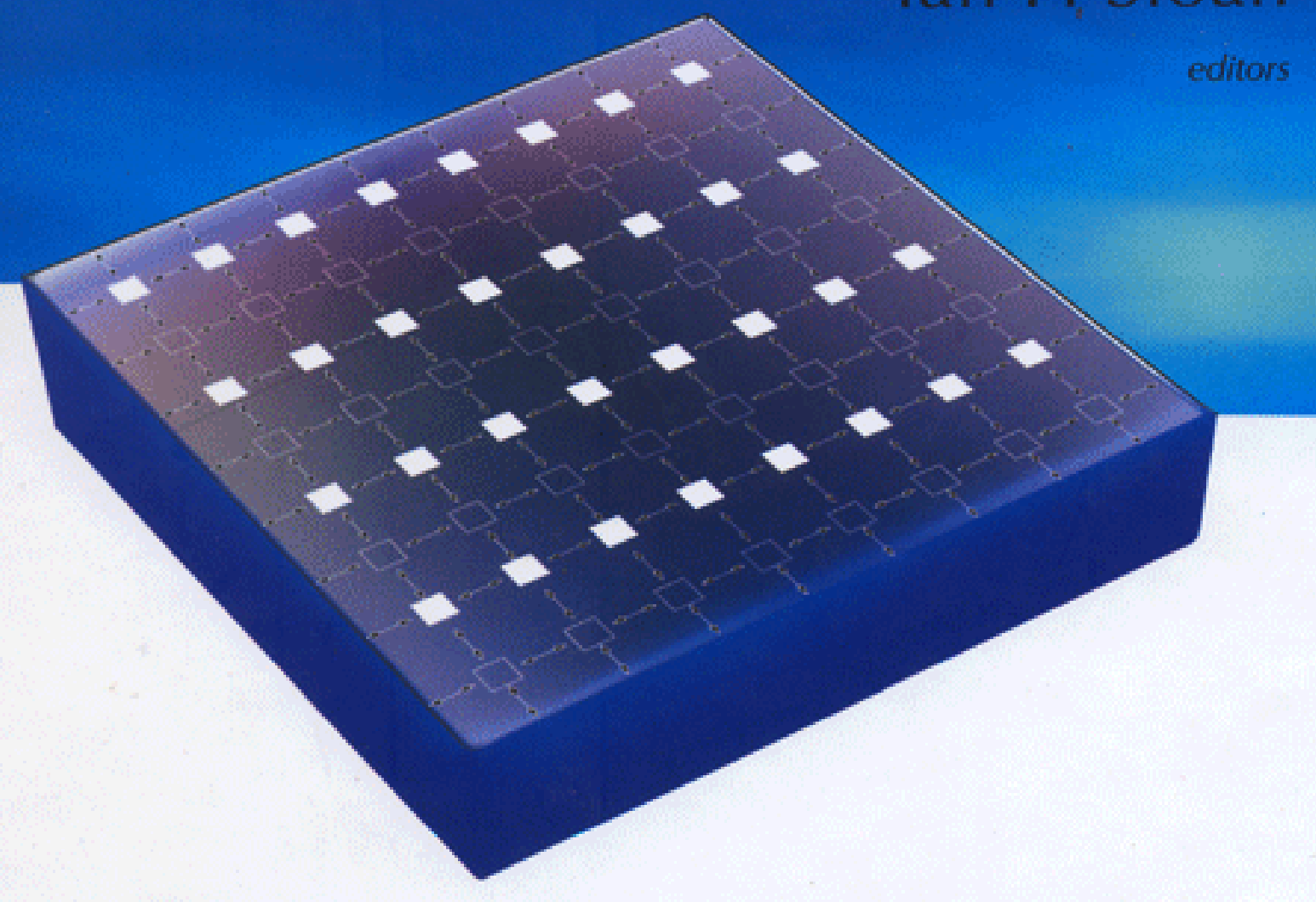




\section{Series in Contemporary Applied Mathematics CAM}

Honorary Editor: Chao-Hao Gu (Fudan University)

Editors: P. G Ciarlet (City University of Hong Kong), Tatsien Li (Fudan University)

1. Mathematical Finance — Theory and Practice (Eds. Yong Jiongmin, Rama Cont)

2. New Advances in Computational Fluid Dynamics - Theory, Methods and Applications

(Eds. F. Dubois, Wu Huamo)

3. Actuarial Science — Theory and Practice

(Eds. Hanji Shang, Alain Tosseti)

4. Mathematical Problems in Environmental Science and Engineering

(Eds. Alexandre Ern, Liu Weiping)

5. Ginzburg-Landau Vortices

(Eds. Haïm Brezis, Tatsien Li)

6. Frontiers and Prospects of Contemporary Applied Mathmetics

(Eds. Tatsien Li, Pingwen Zhang)

7. Mathematical Methods for Surface and Subsurface Hydrosystems

(Eds. Deguan Wang, Christian Duquennoi, Alexandre Ern)

8. Some Topics in Industrial and Applied Mathematics

(Eds. Rolf Jeltsch, Tatsien Li, Ian Hugh Sloan) 
Series in Contemporary Applied Mathematics CAM 8

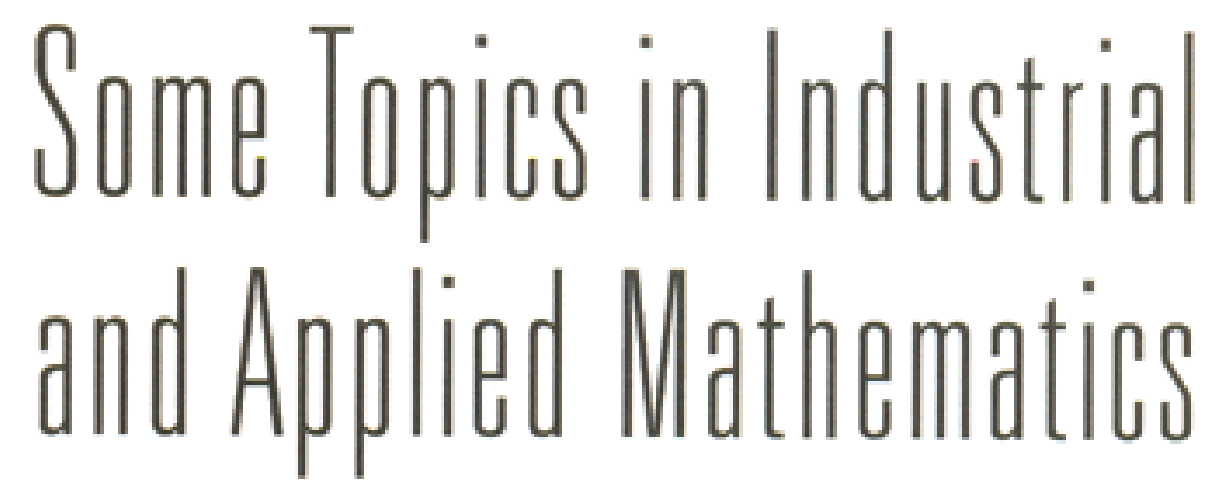

editors

Rolf Jeltsch

ETH Zurich, Switzerland

Ta-Tsien Li

Fudan University, China

Ian H Sloan

University of New South Wales, Australia

Higher Education Press

World Scientific 


$\begin{array}{lll}\text { Rolf Jeltsch } & \text { Tatsien Li } & \text { Ian Hugh Sloan } \\ \text { Seminar of Applied } & \text { School of Mathematical Sciences } & \text { School of Mathematics } \\ \text { Mathematics } & \text { Fudan University } & \text { University of New South Wales } \\ \text { ETH Zürich } & \text { 220, Handan Road } & \text { Sydney NSW 2052 } \\ \text { CH-8092 Zürich } & \text { Shanghai, 200433 } & \text { Australia } \\ \text { Switzerland } & \text { China } & \end{array}$

\section{图书在版编目 (CIP) 数据}

工业与应用数学中的一些问题 $=$ Some Topics in Industrial and Applied Mathematics: 英文 / (瑞士) 杰 尔奇 (Jeltsch, R.), 李大潜, (澳) 斯隆(Sloan, I. H.)

主编. 一北京: 高等教育出版社, 2007.7

(现代应用数学丛书)

ISBN 978-7-04-021903-6

I.工. . I I.(1)杰…(2)李…(3)斯… III.(1)工业工程一应用 数学一英文 IV. TB11

中国版本图书馆 CIP 数据核字 (2007) 第 098434 号

Copyright (C) 2007 by

Higher Education Press

4 Dewai Dajie, Beijing 100011, P. R. China, and

World Scientific Publishing Co Pte Ltd

5 Toh Tuch Link, Singapore 596224

All rights reserved. No part of this book may be reproduced or transmitted in any form or by any means, electronic or mechanical, including photocopying, recording or by any information storage and retrieval system, without permission in writing from the Publisher.

\section{ISBN 978-7-04-021903-6}

Printed in P. R. China 


\section{Contents}

\section{Preface}

Samir Adly, Daniel Goeleven, Michel Théra: A Continuation Method for a Class of Periodic Evolution Variational Inequalities ........ 1 Götz Alefeld: Complementarity Problems: An Overview on

Existing Verification Procedures $\ldots \ldots \ldots \ldots \ldots \ldots, \ldots, \ldots, \ldots, 29$

Fernando Antoneli, Ana Paula S. Dias, Martin Golubitsky, Yunjiao

Wang. Synchrony in Lattice Differential Equations .......... 43 Alain Damlamian: The Periodic Unfolding Method for Quasi-convex

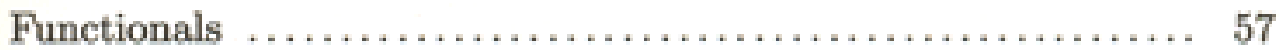

Iain S. Duff: Sparse System Solution and the HSL Library ........ 78 Heinz W. Engl, Gerald Goekler, Andrea Schatz, Helmut Zeisel:

Modelling and Numerics for the Transient Simulation of the Blast Furnace Process $\ldots \ldots \ldots \ldots \ldots \ldots \ldots \ldots \ldots \ldots \ldots . \ldots 5$

Loredana Faienza, Antonio Fasano, Mario Primicerio:

Gelification of Hydrocarbons: A Model Problem

Nguyen Van Hut, Vuong Quan Hoang: On the Martingale

Representation Theorem and on Approximate

Hedging a Contingent Claim in the Minimum

Deviation Square Criterion

Rolf Jeltsch, Manuel Torrilhon: Flexible Stability Domains for

Explicit Runge-Kutta Methods

Taketomo Mitsui, Yoshihiro Saito: MS-Stability Analysis for

Numerical Solutions of Stochastic Differential Equations

-Beyond Single-step Single Dim-

R. D. Russell, J. F. Williams, X. Xu: A Comparison of

Direct Discretization of 4th Order Problems

Versus System Reduction

Ya-xiang Yuan: Subspace Techniques for Nonlinear

Optimization 


\section{Preface}

On the occasion that the Officers' Meeting and the Board Meeting of ICIAM (International Council for Industrial and Applied Mathematics) was held in Shanghai from May 26 to May 27, 2006, many famous industrial and applied mathematicians gathered in Shanghai from different countries. The Shanghai Forum on Industrial and Applied Mathematics was organized from May 25 to May 26, 2006 at Shanghai Science Hall for the purpose of inviting some of them to present their recent results and discuss recent trends in industrial and applied mathematics. Sixteen invited lectures have been given for this activity. This volume collects the material covered by most of these lectures. It will be very useful for graduate students and researchers in industrial and applied mathematics.

The editors would like take this opportunity to express their sincere thanks to all the authors in this volume for their kind contribution. We are very grateful to the Shanghai Association for Science and Technology (SAST), Fudan University, the National Natural Science Foundation of China (NSFC), The China Society for Industrial and Applied Mathematics (CSIAM), the Shanghai Society for Industrial and Applied Mathmatics (SSIAM), the Institut Sino-Français de Mathématiques Appliquées (ISFMA) and the International Council for Industrial and Applied Mathematics (ICIAM) for their help and support. Our special thanks are also due to Mrs. Zhou Chunlian for her efficient assistance in editing this book. 


\title{
On the Martingale Representation Theorem and on Approximate Hedging a Contingent Claim in the Minimum Deviation Square Criterion
}

\author{
Nguyen Van Huu \\ Vietnam National University, Hanoi \\ Vuong Quan Hoang
}

ULB Belgium.

\begin{abstract}
In this work we consider the problem of the approximate hedging of a contingent claim in the minimum mean square deviation criterion. A theorem on martingale representation in case of discrete time and an application of the result for semi-continuous market model are also given.
\end{abstract}

Keywords Hedging, contingent claim, risk neutral martingale measure, martingale representation

\section{Introduction}

The activity of a stock market takes place usually in discrete time. Unfortunately such markets with discrete time are in general incomplete and so super-hedging a contingent claim is requires usually an initial price two great, which is not acceptable in practice.

The purpose of this work is to propose a simple method for approximate hedging a contingent claim or an option in minimum mean square deviation criterion. 


\section{Financial market model with discrete time:}

Without loss of generality let us consider a market model described by a sequence of random vectors $\left\{S_{n}, n=0,1, \cdots, N\right\}, S_{n} \in \mathbb{R}^{d}$, which are discounted stock prices defined on the same probability space $\{\Omega, \Im, P\}$ with $\left\{F_{n}, n=0,1, \cdots, N\right\}$ being a sequence of sigma-algebras of information available up to the time $n$, whereas "risk free" asset chosen as a numeraire $S_{n}^{0}=1$.

A $F_{N}$-measurable random variable $H$ is called a contingent claim (in the case of a standard call option $H=\max \left(S_{n}-K, 0\right), K$ is strike price).

\section{Trading strategy:}

A sequence of random vectors of $d$-dimension $\gamma=\left(\gamma_{n}, n=1,2, \cdots, N\right)$ with $\gamma_{n}=\left(\gamma_{n}^{1}, \gamma_{n}^{2}, \cdots, \gamma_{n}^{d}\right)^{T}\left(A^{T}\right.$ denotes the transpose of matrix $\left.A\right)$, where $\gamma_{n}^{j}$ is the number of securities of type $j$ kept by the investor in the interval $[n-1, n)$ and $\gamma_{n}$ is $F_{n-1}$-measurable (based on the information available up to the time $n-1$ ), then $\left\{\gamma_{n}\right\}$ is said to be predictable and is called Portfolio or trading strategy .

\section{Assumptions:}

Suppose that the following conditions are satisfied:

(i) $\Delta S_{n}=S_{n}-S_{n-1}, H \in L_{2}(P), n=0,1, \cdots, N$.

(ii) Trading strategy $\gamma$ is self-financing, i.e. $S_{n-1}^{T} \gamma_{n-1}=S_{n-1}^{T} \gamma_{n}$ or equivalently $S_{n-1}^{T} \Delta \gamma_{n}=0$ for all $n=1,2, \cdots, N$.

Intuitively, this means that the portfolio is always rearranged in such a way its present value is preserved.

(iii) The market is of free arbitrage, that means there is no trading strategy $\gamma$ such that $\gamma_{1}^{T} S_{0}:=\gamma_{1} S_{0} \leq 0, \gamma_{N} S_{N} \geq 0, P\left\{\gamma_{N} S_{N}>\right.$ $0\}>0$.

This means that with such trading strategy one need not an initial capital, but can get some profit and this occurs usually as the asset $\left\{S_{n}\right\}$ is not rationally priced.

Let us consider

$$
G_{N}(\gamma)=\sum_{k=1}^{N} \gamma_{k} \Delta S_{k} \text { with } \gamma_{k} \Delta S_{k}=\sum_{j=1}^{d} \gamma_{k}^{j} \Delta S_{k}^{j} .
$$


This quanlity is called the gain of the strategy $\gamma$.

The problem is to find a constant $c$ and $\gamma=\left(\gamma_{n}, n=1,3, \cdots, N\right)$ at that

$$
E_{P}\left(H-c-G_{N}(\gamma)\right)^{2} \rightarrow \min .
$$

Problem (1.1) have been investigated by scversl yuthori such as $\mathbf{H}$. Folmer, M Schweiser, M. Schel, M. L. Nechaev with $\alpha=1$. However, the solution of problem (1.1) is very complicated and dillicult for application if $\left\{S_{n}\right\}$ is not a $\left\{\mathbf{F}_{n}\right\}$-martingale under $P$, even for $s=1$.

By the fundamental theorem of firancial mathematics, since the market is of free arbitrage, there cxists a probability measure $Q \sim I^{\prime}$ such that under $Q,\left\{S_{n}\right\}$ is an $\left\{\mathbf{F}_{n}\right\}$-martingnle, i.e. $F_{Q}\left(S_{n} \mid F_{n}\right)=S_{n-1}$ and the measure $Q$ is called risk neutral martingale probability mrasure.

We try to find $c$ and $\gamma$ so lhit

$$
E_{Q}\left(H-c-G_{N}(\gamma j)^{2} \rightarrow \min \text { over } \gamma .\right.
$$

Definition $1.1\left\langle\gamma_{n}^{*}\right\rangle=\left(\gamma_{e}^{*}(c)\right)$ minimizing the expectation in $(1.2)$ + is called $Q$ - optimal stratesy in the minimum mcan equare devistion (MMSD) criterion corresponding to the inilial capital $c$.

The solution of this problem is very simple and the conatriction of the $Q$-optimal strategy is exsy to implement in practicc.

Notice that if $L_{N}-d Q / d P$ then

$$
E_{Q_{\delta}}\left(H-c-G_{N}(\gamma)\right)^{2}=E_{P}\left[\left(H-c-G_{N}\right)^{2} L_{N}\right]
$$

can be considered as an weighted expectulison under $P$ of $\left(H-c-G_{N}\right)^{3}$ with the weight $L_{N}$. This is sirnilar to the pricing asset bused on a ribk neutral martingale measure $Q$.

In this work we give a solution of the problem (1.2) and a theorem on martingalc repreaentation in the case of discrete time.

It is worth to notice that the authors M.Schweiser, M.Sclual: M.L, Nechacv considered only the problem (1.1) with $S_{;}$of one-dimansiou and M.Schweiser necxl the additional assumptions that $\left\{S_{n}\right\}$ satisfies notl-degeneracy condition in the anse that there exists a constant $\delta$ in 
$(0,1)$ such that

$$
\left(E\left[\Delta S_{n} \mid F_{n-1}\right]\right)^{2} \leq \sigma E\left[\left(\Delta S_{n}\right)^{2} \mid F_{n-1}\right] \quad \text { P-a.s. for all } n=1,2, \cdots, N .
$$

and the trading strategies $\gamma_{n}$ 's satisfy:

$$
E\left[\gamma_{n} \Delta S_{n}\right]^{2}<\infty
$$

while in this article $\left\{S_{n}\right\}$ is of $d$-dimension and we need not the preceding assumptions.

The organization of this article is as follows:

The solution of the problem (1.2) is fulfilled in paragraph 2. (Theorem 2.1) and a theorem on the representation of a martingale in terms of the differences $\Delta S_{n}$ (Theorem 3.1) will be also given (the representation is similar to the one of a martingale adapted to a Wiener filter in the case of continuous time).

Some examples are given in paragraph 3.

The semi-continuous model, a type of discretization of diffusion model, is investigated in paragraph 4.

\section{Finding the optimal portfolio}

Notation. Let $Q$ be a probability measure such that $Q$ is equivalent to $P$ and under $Q\left\{S_{n}, n=1,2, \cdots, N\right\}$ is an integrable square martingale and let us denote $E_{n}(X)=E_{Q}\left(X \mid F_{n}\right), H_{N}=H, H_{n}=E_{Q}\left(H \mid F_{n}\right)=$ $E_{n}(H) ; \operatorname{Var}_{n-1}(X)=\left[\operatorname{Cov}_{n-1}\left(X_{i}, X_{j}\right)\right]$ denotes the conditional variance matrix of random vector $X$ when $F_{n-1}$ is given, $\Gamma$ is the family of all predictable strategies $\gamma$.

Theorem 2.1 If $\left\{S_{n}\right\}$ is an $\left\{F_{n}\right\}$-martingale under $Q$ then

$$
E_{Q}\left(H-H_{0}-G_{N}\left(\gamma^{*}\right)\right)^{2}=\min \left\{E_{Q}\left(H-c-G_{N}(\gamma)\right)^{2}: \gamma \in \Gamma\right\},
$$

where $\gamma_{n}^{*}$ is a solution of the following equation system:

$$
\operatorname{lVar}_{n-1}\left(\Delta S_{n}\right) \mid \gamma_{n}^{*}=E_{n-1}\left(\left(\Delta H_{n} \Delta S_{n}\right) \quad\right. \text { P- a.s. }
$$


Proof: At first let us notice that the right side of (2.1) is finite. In fact, with $\gamma_{n}=1$ for all $n$, we have

$$
E_{Q}\left(H-c-G_{N}(\gamma)\right)^{2}=E_{Q}\left(H-c-\sum_{n=1}^{N} \sum_{j=1}^{d} \delta S_{n}^{j}\right)^{2}<\infty .
$$

Furthermore, we shall prove that $\gamma^{*} \Delta S_{n}$ is integrable square under $Q$.

Recall that ( see [Appendix A] ) if $Y, X_{1}, X_{2}, \cdots, X_{d}$ are $d+1$ integrable square random variables with $E(Y)=E\left(X_{1}\right)=\cdots=E\left(X_{d}\right)=0$ and if $\widehat{Y}=b_{1} X_{1}+b_{2} X_{2}+\cdots+b_{d} X_{d}$ is the optimal linear predictor of $Y$ on the basis of $X_{1}, X_{2}, \cdots, X_{d}$ then the vector $b=\left(b_{1}, b_{2}, \cdots, b_{d}\right)^{T}$ is the solution of the following equations system:

$$
\operatorname{Var}(X) b=E(Y X),
$$

and as $\operatorname{Var}(X)$ is non-degenerated $b$ is defined by

$$
b=[\operatorname{Var}(X)]^{-1} E(Y X),
$$

and in all cases

$$
b E(Y X) \leq E\left(Y^{2}\right),
$$

where $X=\left(X_{1}, X_{2}, \cdots, X_{k}\right)^{T}$.

Furthermore,

$$
Y-\widehat{Y} \perp \text {, i.e. } E\left[X_{i}(Y-\widehat{Y})\right]=0, i=1, \cdots, k .
$$

Applying the above results to the problem of conditional linear prediction of $\Delta H_{n}$ on the basis of $\Delta S_{n}^{1}, \Delta S_{n}^{2}, \cdots, \Delta S_{n}^{d}$ as $F_{n}$ is given we obtain from (2.3) the formula (2.2) defining the regression coefficient vector $\gamma^{*}$. On the other hand we have from (2.3) and (2.5):

$$
\begin{aligned}
E\left(\gamma_{n}^{*} \Delta S_{n}\right)^{2} & =E E_{n-1}\left(\gamma_{n}^{*} \Delta S_{n}^{T} \Delta S_{n} \gamma_{n}^{* T}\right)=E\left(\gamma_{n}^{*} \operatorname{Var}_{n-1}\left(\Delta S_{n}\right) \gamma_{n}^{* T}\right) \\
& =E\left(\gamma_{n}^{*} E_{n-1}\left(\Delta H_{n} \Delta S_{n}^{T}\right)\right) \leq E\left(\Delta H_{n}\right)^{2}<\infty .
\end{aligned}
$$

With the above remarks we can consider only, with no loss of generality, trading strategies $\gamma_{n}$ such that

$$
E_{n-1}\left(\gamma_{n} \Delta S_{n}\right)^{2}<\infty
$$


We have:

$$
H_{N}=H_{0}+\Delta H_{1}+\cdots+\Delta H_{N}
$$

and

$$
\begin{aligned}
E_{n-1}\left(\Delta H_{n}-\gamma_{n}^{T} \Delta S_{n}\right)^{2}= & E_{n-1}\left(\Delta H_{n}\right)^{2}-2 \gamma_{n}^{T} E_{n-1}\left(\left(\Delta H_{n} \Delta S_{n}\right)\right. \\
& +\gamma_{n}^{T} E_{n-1}\left(\Delta S_{n} \Delta S_{n}^{T}\right) \gamma_{n} .
\end{aligned}
$$

This expression takes the minimum value when $\gamma_{n}=\gamma_{n}^{*}$.

Furthermore, since $\left\{H_{n}-c-G_{n}(\gamma)\right)$ is an $\left\{F_{n}\right\}$ - integrable square martingale under $Q$,

$$
\begin{aligned}
E_{Q}\left(H_{N}-c-G_{N}(\gamma)\right)^{2}=E_{Q}\left[H_{0}-c-\sum_{n=1}^{N}\left(\Delta H_{n}-\gamma_{n} \Delta S_{n}\right)\right]^{2} \\
=\left(H_{0}-c\right)^{2}+E_{Q}\left[\sum_{n=1}^{N}\left(\Delta H_{n}-\gamma_{n} \Delta S_{n}\right)\right]^{2} \\
=\left(H_{0}-c\right)^{2}+\sum_{n=1}^{N} E_{Q}\left(\Delta H_{n}-\gamma_{n} \Delta S_{n}\right)^{2} \\
\quad\left(\text { for } \Delta H_{n}-\gamma_{n} \Delta S_{n} \text { is martingale difference }\right) \\
=\left(H_{0}-c\right)^{2}+E_{Q} \sum_{n=1}^{N} E_{n-1}\left(\Delta H_{n}-\gamma_{n} \Delta S_{n}\right)^{2} \\
\geq\left(H_{0}-c\right)^{2}+E_{Q} \sum_{n=1}^{N} E_{n-1}\left(\Delta H_{n}-\gamma_{n}^{*} \Delta S_{n}\right)^{2} \\
=\left(H_{0}-c\right)^{2}+E_{Q} \sum_{n=1}^{N}\left(\Delta H_{n}-\gamma_{n}^{*} \Delta S_{n}\right)^{2} \\
=\left(H_{0}-c\right)^{2}+E_{Q}\left[\sum_{n=1}^{N}\left(\Delta H_{n}-\gamma_{n}^{*} \Delta S_{n}\right)\right]^{2} \\
\geq E_{Q}\left(H_{N}-H_{0}-G_{n}\left(\gamma^{*}\right)\right)^{2} .
\end{aligned}
$$

So $E_{Q}\left(H_{N}-c-G_{N}(\gamma)\right)^{2} \geq E_{Q}\left(H_{N}-H_{0}-G_{n}\left(\gamma^{*}\right)\right)^{2}$ and the inequality become the equality if $c=H_{0}$ and $\gamma=\gamma^{*}$. 


\section{Martingale representation theorem}

Theorem 3.1. Let $\left\{H_{n}, n=0,1,2, \cdots\right\},\left\{S_{n}, n=0,1,2, \cdots\right\}$ be arbitrary integrable square random variable defined on the same probability space $\{\Omega, \Im, P\}, F_{n}^{S}=\sigma\left(S_{0}, \cdots, S_{n}\right)$. Denoting by $\Pi(S, P)$ the set of probability measures $Q$ such that $Q \sim P$ and that $\left\{S_{n}\right\}$ is $\left\{F_{n}^{S}\right\}$ integrable square martingale under $Q$, then if $F=\bigvee_{n=0}^{\infty} F_{n}^{S}, H_{n}, S_{n} \in L_{2}(Q)$ and if $\left\{H_{n}\right\}$ is also a martingale under $Q$ we have:

$$
\text { 1. } H_{n}=H_{0}+\sum_{k=1}^{n} \gamma_{k} \Delta S_{k}+C_{n} \quad \text { a.s., }
$$

where $\left\{C_{n}\right\}$ is a $\left\{F_{n}^{S}\right\}-Q$-martingale orthogonal to the martingale $\left\{S_{n}\right\}$, i.e. $E_{n-1}\left(\left(\Delta C_{n} \Delta S_{n}\right)=0\right.$, for all $n=0,1,2, \cdots$, whereas $\left\{\gamma_{n}\right\}$ is $\left\{F_{n-1}^{S}\right\}$ - predictable.

$$
\text { 2. } H_{n}=H_{0}+\sum_{k=1}^{n} \gamma_{k} \Delta S_{k}:=H_{0}+G_{n}(\gamma) \quad \text { P-a.s.. }
$$

for all $n$ finite iff the set $\Pi(S, P)$ consists of only one element.

Proof: According to the proof of Theorem 2.1, putting

$$
\Delta C_{k}=\Delta H_{k}-\gamma_{k}^{*} \Delta S_{k}, C_{n}=\sum_{k=1}^{n} \Delta C_{k}, C_{0}=0,
$$

then $\Delta C_{k} \perp \Delta S_{k}$, by (2.6).

Taking summation of (3.3) we obtain (3.1).

The conclusion 2 follows from the fundamental theorem of financial mathematics.

Notice 1. By the fundamental theorem of financial mathematics a security market has no arbitrage opportunity and is complete iff $\Pi(S, P)$ consists of the only element and in this case we have (3.2) with $\gamma$ defined by (2.2). Furthermore, in this case the conditional probability distribution of $S_{n}$ given $F_{n-1}^{S}$ concentrates at most $d+1$ points of $R^{d}$ (see [2], [3]), in particular for $d=1$, with exception of binomial or generalized binomial market models (see [2], [7]), other models are incomplete.

Notice 2. We can choose the risk neutral martingale probability measure $Q$ so that $Q$ has minimum entropy in $\Pi(S, P)$ as in [2] or $Q$ is near $P$ as much as possible. 
On the Martingale Representation Theorem...

Example 3.1. Let us consider a stock with the discounted price $S_{0}$ at $t=0, S_{1}$ at $t=1$, where

$$
S_{1}= \begin{cases}4 S_{0} / 3 & \text { with prob. } p_{1} \\ S_{0} & \text { with prob. } p_{2}, p_{1}, p_{2}, p_{3}>0, p_{1}+p_{2}+p_{3}=1 \\ 5 S_{0} / 6 & \text { with prob. } p_{3}\end{cases}
$$

Suppose that there is an option on the above stock with the maturity at $t=1$ and with strike price $K=S_{0}$. We shall show that there are several probability measures $Q \sim P$ such that $\left\{S_{0}, S_{1}\right\}$ is, under $Q$, a martingale or equivalently $E_{Q}\left(\Delta S_{1}\right)=0$.

In fact, suppose that $Q$ is a probability measure such that under $Q S_{1}$ takes the values $4 S_{0} / 3, S_{0}, 2 S_{0} / 3$ with positive probability $q_{1}, q_{2}, q_{3}$, respectively. Then $E_{Q}\left(\Delta S_{1}\right)=0 \Leftrightarrow S_{0}\left(q_{1} / 3-q_{3} / 6\right)=0 \Leftrightarrow 2 q_{1}=q_{3}$, so $Q$ is defined by $\left(q_{1}, 1-3 q_{1}, 2 q_{1}\right), 0<q_{1}<1 / 3$.

In the above market, the payoff of the option is

$$
H=\left(S_{1}-K\right)_{+}=\left(\Delta S_{1}\right)_{+}=\max \left(\Delta S_{1}, 0\right) .
$$

It is easy to get a $Q$-optimal portfolio

$$
\begin{gathered}
\gamma^{*}=E_{Q}\left[H \Delta S_{1}\right] / E_{Q}\left(\Delta S_{1}\right)^{2}=2 / 3, E_{Q}(H)=q_{1} S_{0} / 3, \\
E_{Q}\left[H-E_{Q}(H)-\gamma^{*} \Delta S_{1}\right]^{2}=q_{1} S_{0}^{2}\left(1-3 q_{1}\right) / 9 \rightarrow 0 \text { as } q_{1} \rightarrow 1 / 3 .
\end{gathered}
$$

However we can not choose $q_{1}=1 / 3$, because $q=(1 / 3,0,2 / 3)$ is not equivalent to $P$. It is better to choose $q_{1} \cong 1 / 3$ and $0<q_{1}<1 / 3$.

Example 3.2 Let us consider a market with one risky asset defined by:

$$
S_{n}=S_{0} \Pi Z_{i} \text {, or } S_{n}=S_{n-1} Z_{n}, n=1,2, \cdots, N,
$$

where $Z_{1}, Z_{2}, \cdots, Z_{N}$ are the sequence of i.i.d. random variables taking the values in the set $\Omega=\left\{d_{1}, d_{2}, \cdots, d_{M}\right)$ and $P\left(Z_{i}=d_{k}\right)=p_{k}>0, k=$ $1,2, \cdots, M$. It is obvious that a probability measure $Q$ is equivalent to $P$ and under $Q\left\{S_{n}\right\}$ is a martingale if and only if $Q\left\{Z_{i}=d_{k}\right)=q_{k}>$ $0, k=1,2, \cdots, M$ and $E_{Q}\left(Z_{i}\right)=1$, i.e.

$$
q_{1} d_{1}+q_{2} d_{2}+\cdots+q_{M} d_{M}=1 \text {. }
$$


Let us recall the integral Hellinger of two measure $Q$ and $P$ defined on some measurable space $\left\{\Omega^{*}, F\right\}$ :

$$
H(P, Q)=\int_{\Omega^{*}}(d P \cdot d Q)^{1 / 2} .
$$

In our case we have

$$
\begin{gathered}
H(P, Q)=\sum\left\{P\left(Z_{1}=d_{i 1}, Z_{2}=d_{i 2}, \cdots, Z_{N}=d_{i N}\right) .\right. \\
\left.Q\left(Z_{1}=d_{i 1}, Z_{2}=d_{i 2}, \cdots, Z_{N}=d_{i N}\right)\right\}^{1 / 2} \\
=\sum\left\{p_{i 1} q_{i 1} p_{i 2} q_{i 2} \cdots p_{i N} q_{i N}\right\}^{1 / 2},
\end{gathered}
$$

where the summation is extended over all $d_{i 1}, d_{i 2}, \cdots, d_{i N}$ in $\Omega$ or over all $i_{1}, i_{2}, \cdots, i_{N}$ in $\{1,2, \cdots, M\}$. Therefore

$$
H(P, Q)=\left\{\sum_{i=1}^{M}\left(p_{i} q_{i}\right)^{1 / 2}\right\}^{N} .
$$

We can define a distance between $P$ and $Q$ by

$$
\|Q-P\|^{2}=2(1-H(P, Q)) .
$$

Then we want to choose $Q^{*}$ in $\Pi(S, P)$ so that $\left\|Q^{*}-P\right\|=\inf \{\|Q-P\|$ : $Q P(S, P)\}$ by solving the following programming problem:

$$
\sum_{i=1}^{M} p_{i}^{1 / 2} q_{i}^{1 / 2} \rightarrow \max
$$

with the constraints :

a. $q_{1} d_{1}+q_{2} d_{2}+\cdots+q_{M} d_{M}=1$.

b. $q_{1}+q_{2}+\cdots+q_{M}=1$.

c. $q_{1}, q_{2}, \cdots, q_{M}>0$.

Giving $p_{1}, p_{2}, \cdots, p_{M}$ we can obtain a numerical solution of the above programming problem. It is possible that the above problem has not a solution. However, we can replace the condition c) by the condition $\mathrm{c}^{\prime} . q_{1}, q_{2}, \cdots, q_{d} \geq 0$,

then the problem has always the solution $q^{*}=\left(q_{1}^{*}, q_{2}^{*}, \cdots, q_{M}^{*}\right)$ and we can choose the probabilities $q_{1}, q_{2}, \cdots, q_{M}>0$ are sufficiently near to $q_{1}^{*}, q_{2}^{*}, \cdots, q_{M}^{*}$. 


\section{Semi-continuous market model (discrete in time continuous in state )}

Let us consider a financial market with two assets:

+ Free risk asset $\left\{B_{n}, n=0,1, \cdots, N\right\}$ with dynamics

$$
B_{n}=\exp \left(\sum_{k=1}^{n} r_{k}\right), 0<r_{n}<1 .
$$

+ Risky asset $\left\{S_{n}, n=0,1, \cdots, N\right\}$ with dynamics

$$
S_{n}=S_{0} \exp \left(\sum_{k=1}^{n}\left[\mu\left(S_{k-1}\right)+\sigma\left(S_{k-1}\right) g_{k}\right]\right),
$$

where $\left\{g_{n}, n=0,1, \cdots, N\right\}$ is a sequence of i.i.d. normal random variable $N(0,1)$. It follows from (4.2) that

$$
S_{n}=S_{n-1} \exp \left(\mu\left(S_{n-1}\right)+\sigma\left(S_{n-1}\right) g_{n}\right),
$$

where $S_{0}$ is given and $\mu\left(S_{n-1}\right):=a\left(S_{n-1}\right)-2\left(S_{n-1}\right) / 2$, with $a(x), \sigma(x)$ being some functions defined on $[0, \infty)$.

The discounted price of risky asset $\tilde{S}_{n}=S_{n} / B_{n}$ is equal to

$$
\tilde{S}_{n}=S_{0} \exp \left(\sum_{k=1}^{n}\left[\mu\left(S_{k-1}\right)-r_{k}+\sigma\left(S_{k-1}\right) g_{k}\right]\right) .
$$

We try to find a martingale measure $Q$ for this model.

It is easy to see that $E_{P}\left(\exp \left(\lambda g_{k}\right)\right)=\exp \left(\lambda^{2} / 2\right)$, for $g_{k} \sim N(0,1)$, hence

$$
E \exp \left(\sum_{k=1}^{n}\left[\beta_{k}\left(S_{k-1}\right) g_{k}-\beta_{k}\left(S_{k-1}\right)^{2} / 2\right]\right)=1,
$$

for all random variable $\beta_{k}\left(S_{k-1}\right)$.

Therefore, putting

$$
L_{n}=\exp \left(\sum_{k=1}^{n}\left[\beta_{k}\left(S_{k-1}\right) g_{k}-\beta_{k}\left(S_{k-1}\right)^{2} / 2\right]\right), n=1, \cdots, N
$$

and if $Q$ is a measure such that $d Q=L_{N} d P$ then $Q$ is also a probability measure. Furthermore,

$$
\frac{\tilde{S_{n}}}{S_{n-1}^{\tilde{n}}}=\exp \left(\mu\left(S_{n-1}\right)-r_{n}+\sigma\left(S_{n-1}\right) g_{n}\right) .
$$


Denoting by $E^{0}, E$ expectation operations corresponding to $P, Q$, $E_{n}()=.E\left[() \mid. F_{n}^{S}\right]$ and choosing

$$
\beta_{n}=-\left(a\left(S_{n-1}\right)-r_{n}\right) / \sigma\left(S_{n-1}\right)
$$

then it is easy to see that

$$
E_{n-1}\left[\tilde{S_{n}} / S_{n-1}^{\sim}\right]=E^{0}\left[L_{n} \tilde{S_{n}} / S_{n-1}^{\sim} \mid F_{n}^{S}\right] / L_{n-1}=1
$$

which implies that $\left\{\tilde{S}_{n}\right\}$ is a martingale under $Q$.

Furthermore, under $Q, S_{n}$ can be represented in the form

$$
S_{n}=S_{n-1} \exp \left(\left(\mu^{*}\left(S_{n-1}\right)+\sigma\left(S_{n-1}\right) g_{n}^{*}\right)\right.
$$

Where $\mu^{*}\left(S_{n-1}\right)=r_{n}-\sigma^{2}\left(S_{n-1}\right) / 2, g_{n}^{*}=-\beta_{n}+g_{n}$ is Gaussian $N(0,1)$. It is not easy to show the structure of $\Pi(S, P)$ for this model.

We can choose a such probability measure $Q$ or the weight function $L_{N}$ to find a $Q$ - optimal portfolio.

Notice 3. The model (4.1), (4.2) is a type of discretization of the following diffusion model:

Let us consider a financial market with continuous time consisting of two assets:

+Free risk asset:

$$
B_{t}=\exp \left(\int_{0}^{t} r(u) d u\right)
$$

+Risky asset : $d S_{t}=S_{t}\left[a\left(S_{t}\right) d t+\sigma\left(S_{t}\right) d W t\right], S_{0}$ is given, where $a(),. \sigma():.(0, \infty) \rightarrow R$ such that $x a(x), x \sigma(x)$ are Lipschitz. It is obvious that

$$
S_{t}=\exp \left\{\int_{0}^{t}\left[a\left(S_{u}\right)-\sigma^{2}\left(S_{u}\right) / 2\right] d u+\int_{0}^{t} \sigma\left(S_{u}\right) d W_{u}\right\}, 0 \leq t \leq T .
$$

Putting

$$
\mu(S)=a(S)-\sigma^{2}(S) / 2,
$$


and dividing $[0, T]$ into $N$ intervals by the equidistant dividing points $0, \Delta, 2 \Delta, \cdots, N \Delta$ with $N=T / \Delta$ sufficiently great, it follows from (4.10), (4.11) that

$$
\begin{aligned}
S_{n \Delta} & =S_{(n-1) \Delta} \exp \left\{\int_{(n-1) \Delta}^{n \Delta} \mu\left(S_{u}\right) d u+\int_{(n-1) \Delta}^{n \Delta} \sigma\left(S_{u}\right) d W_{u}\right\} \\
& \cong S_{(n-1) \Delta} \exp \left\{\mu\left(S_{(n-1) \Delta}\right) \Delta+\left(S_{(n-1) \Delta}\right)\left[W_{n \Delta}-W_{(n-1) \Delta}\right]\right\} \\
& \cong S_{(n-1) \Delta} \exp \left\{\left(S_{(n-1) \Delta}\right) \Delta+\sigma\left(S_{(n-1) \Delta}\right) \Delta^{1 / 2} g_{n}\right\}
\end{aligned}
$$

with $g_{n}=\left[W_{n \Delta}-W_{(n-1) \Delta}\right] / \Delta^{1 / 2}, n=1, \cdots, N$, being a sequence of the i.i.d. normal random variables of the law $N(0,1)$, so we obtain the model:

$$
S_{n \Delta}^{*}=S_{(n-1) \Delta}^{*} \exp \left\{\left(S_{(n-1) \Delta}\right) \Delta+\left(S_{(n-1) \Delta}\right) \Delta^{1 / 2} g_{n}\right\} .
$$

Similarly we have

$$
B_{n \Delta}^{*} \cong B_{(n-1) \Delta}^{*} \exp \left(r_{(n-1) \Delta} \Delta\right) .
$$

According to (4.10), the discounted price of the stock $S_{t}$ is

$$
\tilde{S}_{t}=\frac{S_{t}}{B_{t}}=S_{0} \exp \left\{\int_{0}^{t}\left[\mu\left(S_{u}\right)-r_{u}\right] d_{u}+\int_{0}^{t} \sigma\left(S_{u}\right) d W_{u}\right\} .
$$

By Girsanov Theorem, the unique probability measure $Q$ under which $\left\{\tilde{S}_{t}, F_{t}^{S}, Q\right\}$ is a martingale is defined by

$$
(d Q / d P) \mid F_{T}^{S}=\exp \left(\int_{0}^{T} \beta_{u} d W_{u}-\frac{1}{2} \int_{0}^{T} \beta_{u}^{2} d_{u}\right):=L_{T}(\omega),
$$

where

$$
\beta_{s}=-\frac{\left(\left(a\left(S_{s}\right)-r_{s}\right)\right.}{\sigma\left(S_{s}\right)},
$$

and $(d Q / d P) \mid F_{T}^{S}$ denotes the Radon-Nikodym derivative of $Q$ w.r.t. $P$ limited on $F_{T}^{S}$. Furthermore, under $Q$

$$
W_{t}^{*}=W_{t}+\int_{0}^{t} \beta_{u} d u
$$


is a Wiener process. It is obvious that $L_{T}$ can be approximated by

$$
L_{N}:=\exp \left(\sum_{k=1}^{N} \beta_{k} \Delta^{1 / 2} g_{k}-\Delta \beta_{k}^{2} / 2\right),
$$

where

$$
\beta_{n}=-\frac{\left[a\left(S_{(n-1) \Delta}\right)-r_{n \Delta}\right]}{\sigma\left(S_{(n-1) \Delta}\right)} .
$$

Therefore the weight function (4.14) is approximate to Radon-Nikodym derivative of the risk unique neutral martingale measure $Q$ w.r.t. $P$ and $Q$ is used to price derivatives of the market.

Notice 4. In the market model Black- Scholes we have $L_{N}=L_{T}$. We want to show now that for the weight function (4.17)

$$
E_{Q}\left(H-H_{0}-G_{N}\left(\gamma^{*}\right)\right)^{2} \rightarrow 0 \text { as } N \rightarrow \infty \text { or } \Delta \rightarrow 0 \text {. }
$$

Proposition Suppose that $H=K\left(S_{T}\right) / B_{N}$ is a integrable square discounted contingent claim. Then

$$
E_{Q}\left(H-H_{0}-G_{N}\left(\gamma^{*}\right)\right)^{2} \rightarrow 0 \text { as } N \rightarrow \infty \text { or } \Delta \rightarrow 0,
$$

provided $a$ and $\sigma$ are constant (in this case the model (4.10), (4.11) is the model Black-Scholes).

Proof: It is well known (see[4],[5]) that for the model of complete market (4.10), (4.11) there exists a trading strategy $\varphi=\left(\varphi_{t}=\right.$ $\varphi(t, S(t)), 0=t=T)$, hedging $H$, where $\varphi:[0, T] \times(0, \infty) \rightarrow R$ is continuously derivable in $t$ and $S$, such that

$$
H\left(S_{T}\right)=H_{0}+\int_{0}^{T} \varphi_{t} d \tilde{S}(t) \quad \text { a.s. }
$$


On the other hand we have

$$
\begin{aligned}
& E_{Q_{N}}\left(H-H_{0}-\sum_{k=1}^{N} \gamma_{(k-1) \Delta}^{*} \Delta \tilde{S}_{n \Delta}\right)^{2} \\
& \leq E_{Q_{N}}\left(H-H_{0}-\sum_{k=1}^{N} \phi_{(k-1) \Delta} \Delta \tilde{S}_{n \Delta}\right)^{2} \\
& =E_{Q}\left(\int_{0}^{T} \phi_{t} d \tilde{S}(t)-\sum_{k=1}^{N} \phi_{(n-1) \Delta} \Delta \tilde{S}_{(n-1) \Delta}\right)^{2} L_{N} / L_{T} \\
& =E_{Q}\left(\int_{0}^{T} \phi_{t} d \tilde{S}(t)-\sum_{k=1}^{N} \phi_{(k-1) \Delta} \Delta \tilde{S}_{(n-1) \Delta}\right)^{2} \rightarrow 0 \text { as } \Delta \rightarrow 0 .
\end{aligned}
$$

(Since $L_{N}=L_{T}$ and by the definition of the stochastic integral Ito as a and $\sigma$ are constant.)

\section{A Appendix A}

Let $Y, X_{1}, X_{2}, \cdots, X_{d}$ be integrable square random variables defined on the same probability space $\{\Omega, F, P\}$ such that $E X_{1}=\cdots=E X_{d}=$ $E Y=0$.

We try to find a coefficient vector $b=\left(b_{1}, \cdots, b_{d}\right)^{T}$ so that

$$
E\left(Y-b_{1} X_{1}-\cdots-b_{d} X_{d}\right)^{2}=E\left(Y-b^{T} X\right)^{2}=\min _{a \in R^{d}}\left(Y-a^{T} X\right)^{2} .
$$

Let us denote $E X=\left(E X_{1}, \cdots, E X_{d}\right), \operatorname{Var}(X)=\left[\operatorname{Cov}\left(X_{i}, X_{j}\right), i, j=\right.$ $1,2, \cdots, d]=E X X^{T}$.

Proposition The vector $b$ minimizing $E\left(Y-a^{T} X\right)^{2}$ is a solution of the following equation system:

$$
\operatorname{Var}(X) b=E(X Y) .
$$


Putting $U=Y-b^{T} X=Y-\hat{Y}$, with $\hat{Y}=b^{T} X$, then

$$
\begin{aligned}
& E\left(U^{2}\right)=E Y^{2}-b^{T} E(X Y) \geq 0 . \\
& E\left(U X_{i}\right)=0 \text { for all } i=1, \cdots, d . \\
& E Y^{2}=E U^{2}+E \hat{Y}^{2} . \\
& \rho=\frac{E \hat{Y}^{2}}{\left(E Y^{2} E \hat{Y}^{2}\right)^{1 / 2}}=\left(\frac{E \hat{Y}^{2}}{E Y^{2}}\right)^{1 / 2} .
\end{aligned}
$$

( $\rho$ is called multiple correlation coefficient of $Y$ relative to $X$.)

Proof: Suppose at first that $\operatorname{Var}(X)$ is a positively definite matrix. For each $a \in R^{d}$ We have

$$
\begin{gathered}
F(a)=E\left(Y-a^{T} X\right)^{2}=E Y^{2}-2 a^{T} E(X Y)+a^{T} E X X^{T} a \\
F(a)=-2 E(X Y)+2 \operatorname{Var}(X) a . \\
{\left[\frac{\partial F(a)}{\partial a_{i} \partial a_{j}}, i, j=1,2, \cdots, d\right]=2 \operatorname{Var}(\mathrm{X}) .}
\end{gathered}
$$

It is obvious that the vector b minimizing $F(a)$ is the unique solution of the following equation:

$$
F(a)=0 \text { or }(\mathrm{A} .2)
$$

and in this case (A.2) has the unique solution:

$$
b=[\operatorname{Var}(X)]^{-1} E(X Y) .
$$

We assume now that $1 \leq \operatorname{Rank}(\operatorname{Var}(X))=r<d$.

We denote by $e_{1}, e_{2}, \cdots, e_{d}$ the ortho-normal eigenvectors w.r.t. the eigenvalues $\lambda_{1}, \lambda_{2}, \cdots, \lambda_{d}$ of $\operatorname{Var}(X)$, where $\lambda_{1} \geq \lambda_{2} \geq \cdots \geq \lambda_{r}>0=$ $\lambda_{r+1}=\cdots=\lambda_{d}$ and $P$ is a orthogonal matrix with the columns being the eigenvectors $e_{1}, e_{2}, \cdots, e_{d}$, then we obtain:

$$
\operatorname{Var}(X)=P \Lambda P^{T}, \text { with } \Lambda=\operatorname{Diag}\left(\lambda_{1}, \lambda_{2}, \cdots, \lambda_{d}\right) .
$$

Putting

$$
Z=P^{T} X=\left[e_{1}^{T} X, e_{2}^{T} X, \cdots, e_{d}^{T} X\right]^{T},
$$


$Z$ is the principle component vector of $X$, we have

$$
\operatorname{Var}(Z)=P^{T} \operatorname{Var}(X) P=\Lambda=\operatorname{Diag}\left(\lambda_{1}, \lambda_{2}, \cdots, \lambda_{r}, 0, \cdots, 0\right) .
$$

Therefore

$$
E Z_{r+1}^{2}=\cdots=E Z_{d}^{2}=0 \text {, so } Z_{r+1}=\cdots=Z_{d}=0 \text { P- a.s. }
$$

Then

$$
\begin{aligned}
F(a) & =E\left(Y-a^{T} X\right)^{2}=E\left(Y-\left(a^{T} P\right) Z\right)^{2} \\
& =E\left(Y-a_{1}^{*} Z_{1}-\cdots-a_{d}^{*} Z_{d}\right)^{2} \\
& =E\left(Y-a_{1}^{*} Z_{1}-\cdots-a_{r}^{*} Z_{r}\right)^{2}
\end{aligned}
$$

where

$a^{* T}=\left(a_{1}^{*}, \cdots, a_{d}^{*}\right)=a^{T} P, \quad \operatorname{Var}\left(Z_{1}, \cdots, Z_{r}\right)=\operatorname{Diag}\left(\lambda_{1}, \lambda_{2}, \cdots, \lambda_{r}\right)>0$.

According to the above result $\left(b_{1}^{*}, \cdots, b_{r}^{*}\right)^{T}$ minimizing $E\left(Y-a_{1}^{*} Z_{1}-\right.$ $\left.\cdots-a_{r}^{*} Z_{r}\right)^{2}$ is the solution of

$$
\left(\begin{array}{ccc}
\lambda_{1} & \ldots & 0 \\
\ldots & \ldots & \ldots \\
0 & \ldots & \lambda_{r}
\end{array}\right)\left(\begin{array}{c}
b_{1}^{*} \\
\ldots \\
b_{r}^{*}
\end{array}\right)=\left(\begin{array}{c}
E Z_{1} Y \\
\ldots \\
E X_{r} Y
\end{array}\right)
$$

or

$$
\left(\begin{array}{cccccc}
\lambda_{1} & \ldots & 0 & 0 & \ldots & 0 \\
\ldots & \ldots & \ldots & \ldots & \ldots & \ldots \\
0 & \ldots & \lambda_{r} & 0 & \ldots & 0 \\
0 & \ldots & 0 & 0 & \ldots & 0 \\
\ldots & \ldots & \ldots & \ldots & \ldots & \ldots \\
0 & \ldots & 0 & 0 & \ldots & 0
\end{array}\right)\left(\begin{array}{c}
b_{1}^{*} \\
\ldots \\
b_{r}^{*} \\
b_{r+1}^{*} \\
\ldots \\
b_{d}^{*}
\end{array}\right)=\left(\begin{array}{c}
E Z_{1} Y \\
\ldots \\
E Z_{r} Y \\
0 \\
\ldots \\
0
\end{array}\right)=\left(\begin{array}{c}
E Z_{1} Y \\
\ldots \\
E Z_{r} Y \\
E Z_{r+1} Y \\
\ldots \\
E Z_{d} Y
\end{array}\right)
$$

with $b_{r+1}^{*}, \cdots, b_{d}^{*}$ arbitrary.

Let $b=\left(b_{1}, \cdots, b_{d}\right)^{T}$ be the solution of $b^{T} P=b^{* T}$, hence $b=P b^{*}$ with $b^{*}$ being a solution of (A.9). Then it is follows from (A.9) that

$$
\operatorname{Var}(Z) P^{T} b=E(Z Y)=P^{T} E(X Y)
$$


or

$$
P^{T} \operatorname{Var}(X) P P^{T} b=P^{T} E(X Y)\left(\text { since } \operatorname{Var}(Z)=P^{T} \operatorname{Var}(X) P\right)
$$

or

$$
\operatorname{Var}(X) b=E(X Y)
$$

which is (A.2). Thus we have proved that (A.2) has always a solution, which solves the problem (A.1).

By (A.7), we have

$$
\begin{aligned}
F(b) & =\min _{a} E\left(Y-a_{T} X\right)^{2} \\
& =E Y^{2}-2 b^{T} E(X Y)+b^{T} \operatorname{Var}(X) b \\
& =E Y^{2}-2 b^{T} E(X Y)+b^{T} E(X Y) \\
& =E Y^{2}-b^{T} E(X Y) \geq 0 .
\end{aligned}
$$

On the other hand

$$
E U X_{i}=E\left(X_{i} Y\right)-E\left(X_{i} b^{T} X\right)=0,
$$

since $b$ is a solution of (A.2) and (A.10) is the ith equation of the system (A.2).

It follows from (A.10) that $E(U \hat{Y})=0$ and $E Y^{2}=E(U+\hat{Y})^{2}=E U^{2}+E \hat{Y}^{2}+2 E(U \hat{Y})=E U^{2}+E \hat{Y}^{2}$.

Example We can use Hilbert space method to prove the above proposition. In fact, let $H$ be the set of all random variables $\xi$ 's such that $E \xi=0, E \xi^{2}<\infty$, then $H$ becomes a Hilbert space with the scalar product $(\xi, \zeta)=E \xi \zeta$, and with the norm $\|\xi\|=\left(E \xi^{2}\right)^{1 / 2}$. Suppose that $X_{1}, X_{2}, \cdots, X_{d}, Y \in H, L$ is the linear manifold generated by $X_{1}, X_{2}, \cdots, X_{d}$. We want to find a $\hat{Y} \in H$ so that $\|Y-\hat{Y}\|$ minimizes, that means $\hat{Y}=b^{T} X$ solves the problem (A.1). It is obvious that $\hat{Y}$ is defined by

$$
\hat{Y}=\operatorname{Proj}_{L} Y=b^{T} X \text { and } U=\hat{Y}-Y \in L .
$$

Therefore $\left(Y-b^{T} X, X_{i}\right)=0$ or $E\left(b^{T} X X_{i}\right)=E\left(X_{i} Y\right)$ for all $i=1, \cdots, d$ or $b^{T} E\left(X^{T} X\right)=E(X Y)$ which is the equation (A.2). The rest of the above proposition is proved similarly. 


\section{References}

[1] Follmer H.,Schweiser M. Hedging of contingent claim under incomplete information. App.Stochastic Analysis, Edited by M.Davisand, R.Elliot, London, Gordan\&Breach, 1999, pp.389-414.

[2] Follmer H., Schied A. Stochastic Finance. An introduction in discrete time. Walter de Gruyter, Berlin- New York, 2004.

[3] Jacod J., Shiryaev A.N. Local martingales and the fundamental asset pricing theorem in the discrete case. Finance Stochastic 2, pp. 259-272.

[4] Harrison, M.J. and Kreps, D.M.,Martingales and arbitrage in multiperiod securities markets, J. of Economic Theory, 29 (1979), pp. 381-408.

[5] Harrison, M.J.and Pliska S.R., Martingales and stochastic integrals in theory of continuous trading, Stochastic Processes and their Applications, 11 (1981), pp.216-260.

[6] Lamberton, D. and Lapayes,B. Introduction to Stochastic Calculus Applied in Finance. Chapman\&Hall/CRC, 1996

[7] Nechaev M.L. On mean-Variance hedging. Proceeding of Workshop on Math. Finance, May 18-19, 1998. Institute Franco-Russe Liapunov, Ed. by A.Shiryaev, A. Sulem.

[8] Nguyen Van Huu, Tran Trong Nguyen. On a generalized Cox-RossRubinstein option market model. Acta Math. Vietnamica, 26 (2001), No2,187-204.

[9] Schweiser M. Variance -optimal hedging in discrete time. Mathematics of Operation Research, 20 (1995), pp.1-32.

[10] Schweiser M.Approximation pricing and the variance-optimal martingale measure. The Annals of Prob. 24(1966), 1, pp.206-236.

[11] Schal M.On quadratic cost criteria for option hedging. Mathematics of Operation Research, 19(1994), pp. 131-141. 